\title{
Brevísimas notas sobre Violencia en cinco ciudades colombianas a finales del siglo XX Y principios del siglo XXI
}

ÁLVARO GUZMÁN BARNEY. (ed.) (2018). Violencia en cinco ciudades Colombianas, a finales del siglo XX y principios del siglo $X X I$. Cali: Universidad Autónoma de Occidente, 811 pp.

Basados en el marco conceptual propuesto por Álvaro Camacho y Álvaro Guzmán en su libro pionero Colombia: ciudad y violencia (1990), los distintos autores de Violencia en cinco ciudades colombianas a finales del siglo XX y principios del siglo XXI presentan un diagnóstico impresionante de las múltiples fases de la violencia en Colombia en los últimos treinta años (1980-2012). El libro reúne un equipo multidisciplinario de investigadores/as cubriendo las cinco ciudades más importantes (Bogotá, Medellín, Cali, Barranquilla y Buenaventura) en cinco capítulos y 811 páginas. ¿Qué hizo que Medellín pasara de ser la ciudad más violenta de Colombia en los años 90 a la «tensa calma» de la primera década del siglo XXI? ¿Cuál es la relación entre violencia política y violencia callejera en las periferias urbanas de Bogotá? ¿Cómo los habitantes de los barrios populares de Cali recuerdan los «tiempos de la violencia política», qué visión tienen del Estado y cuál es el impacto del cartel de Cali en la estructura paramafiosa actual? ¿Por qué Barranquilla y Buenaventura, dos ciudades-puerto, tienen trayectorias de violencia tan distintas? ¿Cuál es el papel de las élites económicas en la economía política de la violencia en la Colombia de inicios del siglo XXI?

Para contestar estas y otras preguntas, las/los investigadores rescatan un problema teórico planteado por Guzmán y Camacho en su libro de 1990: ¿cómo entender la violencia urbana en Colombia más allá de la clave «política» privilegiada por los estudios tradicionales de los/las violentólogos/as (una clave que terminaba por 
reducir el problema a las disputas bipartidistas y por sugerir una cultura de la violencia, o un ethos nacional violento)? Su imaginación sociológica nos llevó entonces a una mirada comprensiva y multifacética del fenómeno a partir del concepto-clave de «escenarios de violencia». Algunos de estos escenarios identificados en aquel entonces, y al parecer vigentes hoy en día, son la violencia política (confrontación armada entre fuerzas insurgentes y el Estado), la violencia estructural/económica (el abandono del Estado y las opresiones de clase), la violencia delictiva callejera (con su expresión máxima en las pandillas urbanas) y la violencia intrafamiliar (que se interseca con las anteriores). Todos estos escenarios se concatenan, se articulan y se realimentan en la conformación de un orden social específico. Orden social y escenarios de violencia son interdependientes y constitutivos uno del otro. Por ejemplo, en Cali, hasta los años 1970, las élites conformaron un orden hegemónico filantrópico que facilitaba el control del Estado y de los recursos económicos por medio de la administración calculada de la pobreza y la disimulación de la dominación por medio de la caridad. La violencia era controlada por las élites agrícolas e industriales del Valle por medio del ejercicio autoritario y benevolente del poder. A partir de los años 1980, «la forma de dominación cambia su naturaleza», dicen los autores, «y el orden social se reproduce a partir de una hegemonía paramafiosa» (629). Esta violencia paramafiosa ocupa todos los espacios de la vida urbana, desde las disputas político-partidarias (parapolítica) hasta la violencia pandillera, las relaciones intrafamiliares (el feminicidio como una de sus expresiones) y las violencias callejeras (como el atraco y las riñas).

En Medellín, los autores identifican algo semejante en las dinámicas de la violencia en la ciudad: el proceso de modernización en los años 1970-80 da paso a un orden urbano autoritario y excluyente que facilitará el control de la ciudad por las élites y las estructuras paramafiosas en las décadas siguientes. De la conflictividad asociada al uso y apropiación del suelo, a las redes de negocios ilícitos del control del narcotráfico, la confrontación insurgencia/contrainsurgencia (y las limpiezas sociales establecidas por estos grupos en el espacio urbano marginal), las confrontaciones pandilleras por dominio territorial micro y las luchas populares por el derecho a la ciudad, todos estos escenarios estructuran un modelo muy particular de gobernanza urbana.

Aunque cada ciudad tenga sus dinámicas y trayectorias históricas propias, es posible identificar un continuum, aunque en grados distintos, en Bogotá y Barranquilla, donde el orden urbano injusto y autoritario es el escenario de la lucha por el derecho a la ciudad y el control de los territorios marginados por grupos armados. Las periferias urbanas aparecen en Violencia en cinco ciudades colombianas como una geografia-problema, un territorio en disputa por la soberanía. Pero al contrario de lo 
que podría suponerse, los ejemplos sugieren un orden urbanístico funcional. Tal vez el caso más emblemático de estas articulaciones perversas entre urbanización precaria, abandono estatal, control de la población por parte de actores armados, mercantilización del suelo urbano y orden urbano lucrativo/funcional sea el caso de Buenaventura, tratado en el capítulo IV.

Buenaventura llama la atención por el espectáculo de la muerte (las tristemente famosas casas de pique), pero, en general, en las cinco ciudades analizadas la articulación macabra entre violencia económica, violencia política y control de la vida urbana invita a pensar la instrumentalidad de la violencia en la producción de un régimen neoliberal de gobernanza urbana que favorece no solamente a los actores armados en disputa, sino también a las élites y clases medias (blanco/mestizas) urbanas. Es decir, la violencia no es un «problema» de falta de gobierno; la violencia produce la ciudad neoliberal. Dicho de otra forma, la ciudad es producida en un proceso dialéctico de conflictividades, violencias y articulaciones políticas que producen una forma específica de gobierno, que en el periodo analizado coincide con políticas neoliberales (para Buenaventura, ver por ejemplo el impacto de la privatización del puerto en la escalada de violencias).

Si, como dicen los autores, «la violencia hay que interpretarla a la luz de los conflictos en la sociedad y de los procesos en que se adquiere y retiene poder sobre los otros» (24), entonces se puede argumentar que las geografías macabras producidas por la violencia también resultan funcionales para la constitución y mantenimiento del orden urbano. ¿Estaríamos hablando entonces de un régimen de gobernanza a la vez necro y bio-político (un régimen que crea una forma específica de vida urbana, que sostiene el poder de las élites, que demarca las fronteras de lo legal/ilegal, en una palabra, que produce Estado)?

Si bien en algunos de los capítulos (ver especialmente el dedicado a Cali) hay un referente teórico que sitúa al Estado como producto histórico de relaciones de poder (Tilly, 1985), la pregunta anterior desafía e invita a los/las autoras a reconsiderar una cierta tendencia a analizar el papel del Estado como «disfuncional», «de baja legitimidad» o de «poca presencia» cuando, en realidad, los datos sugieren una manera deliberada del Estado de hacerse presente por medio de la ausencia o de la presencia delincuencial de sus fuerzas de seguridad, como lo ha planteado, por ejemplo, María Clemencia Ramírez (2001) para el caso del Putumayo, donde los campesinos quedan atrapados/as en las disputas entre guerrilla/Estado-paramilitares, y también como lo han denunciado los/las activistas del paro cívico de Buenaventura, para quienes la relación entre el Estado y esta población racializada es una relación de antagonismo y abandono; en definitiva, un abandono funcional para la economía portuaria y para las transacciones narco-mafiosas. Uno de los méritos del libro Vio- 
lencia en cinco ciudades colombianas es mostrar cómo el Estado tiene una capacidad impresionante de repetir errores a lo largo de las décadas analizadas -el estudio presenta un diagnóstico sobre los desastres de las políticas públicas de seguridad en el periodo-, aunque la discusión sobre cómo dichos desastres son constitutivos de la formación estatal en estas geografías de precariedad se queda corta. Asimismo, ¿cómo una mirada más distanciada del Estado como el tipo ideal de justicia y orden nos ayudaría a entender y proponer otras alternativas a la violencia?

El tratamiento dado a las pandillas, por ejemplo, es sintomático de esta mirada Estado-céntrica. El científico David Brotherton (2008) argumenta que, si bien los estudios sobre este fenómeno han ido más allá de las teorías criminógenas sobre la desviación social y han considerado las pandillas como una forma de organización política, como comunidad imaginada o como forma de reproducción social (ver por ejemplo Bourgois, 2003 y Valverde, 2015), las prácticas de los jóvenes pandilleros son raramente vistas como una respuesta política capaz de producir una agencia colectiva más amplia. Aun cuando los pandilleros son considerados sujetos políticos, argumenta Brotherton, sus prácticas son vistas como «oposición sin la posibilidad de cualquier trascendencia política o cultural» capaz de cuestionar los presupuestos políticos de la sociedad (2008: 61). Algunas intervenciones importantes han analizado las pandillas como un fenómeno que va más allá de las formas instrumentales/ pragmáticas de resistencia cotidiana -«everyday forms of resistance» (Scott, 1986)-. Por ejemplo, en América Central, Dennis Rogers (2006) y Lirio Gutiérrez Rivera (2010) han desarrollado una lectura de las pandillas como una forma de gobierno/ gubernamentalidad. Para el contexto de Cali y Sao Paulo, yo mismo he intentado entender las pandillas más allá del paradigma Estado-céntrico y he considerado el mensaje político del rechazo a ser gobernado por los proyectos de disciplina espacial como «policía por cuadrante» $\mathrm{y}$ «policía comunitaria», los proyectos filantrópicos de gestión de la pobreza, las misiones evangélicas y más recientemente la parafernalia de talleres de construcción de paz. He argumentado, por ejemplo, sobre la importancia de una sensibilidad etnográfica para comprender las pandillas como una forma de agencia criminal/espacial, aunque controversial y violenta (Alves 2017 y 2014).

¿En qué medida una mirada más allá del paradigma Estado-céntrico/legal nos posibilita ver otras formas de vida urbana que rechazan el orden político que busca crear sujetos disciplinados? Asimismo, ¿qué otras posibles interpretaciones podríamos ofrecer sobre las pandillas urbanas teniendo en cuenta que este no es un fenómeno solo de la juventud empobrecida? Si los hallazgos de Camacho y Guzmán en los años 1990 siguen siendo válidos (y un hallazgo importante fue llamar la atención de la comunidad académica sobre las interfaces entre violencia política, económica, callejera, etc.), mi llamado es por la politización de la violencia pandillera en escena- 
rios de colonización del territorio por una élite blanco/mestiza perversa, por economías para-legales gore (Valencia, 2012) y por un Estado históricamente delincuente. Como objeto etnográfico, pienso que las pandillas de jóvenes podrían ser un texto que nos ayudaría a entender una cierta continuidad perversa entre las estrategias de gobierno adoptadas por las élites agroindustriales en los años 60-70, la hegemonía paramafiosa de los años 1990-2000 y las nuevas guerras urbanas del posacuerdo.

En efecto, la Colombia urbana del posacuerdo ofrece nuevos desafíos de investigación y el libro Violencia en cinco ciudades colombianas representa una contribución indudablemente singular a este nuevo momento. En este sentido, el referente teórico-conceptual de «escenarios y campos de violencia» permite pensar una economía política de la violencia resultante de relaciones de dominación política, económica y social. Esta es una contribución que debemos a los Álvaros, quienes abrieron el camino con Colombia: ciudady violencia. ¿Entre tanto, será este referente creado hace treinta años todavía válido para entender las nuevas lógicas de la violencia urbana en el siglo XXI? Dejo esta pregunta no para invalidar los hallazgos, sino para invitar a un diálogo productivo con otros enfoques teórico-metodológicos de este prolífico campo de investigación. Los/las lectoras se beneficiarán enormemente de futuros trabajos comparados que expandan los hallazgos presentados de manera aislada en cada capítulo del libro. Imagino que Álvaro Guzmán y su equipo explorarán el rico material que quedó por fuera de la publicación. Asimismo, investigadores/as interesados en establecer relaciones comparativas con otros campos de violencia y otras geografías latinoamericanas encontrarán en el libro un aporte obligatorio para entender las configuraciones históricas, las dinámicas locales y sus articulaciones nacionales/internacionales. De especial importancia, me parece, es la creación de redes de estudios que demuestren el papel del «orden paramafioso» en la constitución de la vida urbana en contextos aparentemente disímiles (como el paramilitarismo en Rio de Janeiro, las políticas de contención de pandillas en Centroamérica, las disputas por soberanía y el monopolio de la violencia homicida en Sao Paulo, o el narcotráfico y las políticas estatales de represión en México). Nuevas líneas de investigación podrán beneficiarse del foco de luz lanzado por Violencia en cinco ciudades colombianas.

Por último, una breve consideración sobre las cuestiones identitarias: el libro presenta una muy bienvenida y muy esperada discusión sobre la racialización de la violencia urbana en Cali (y de manera poco detenida, en Buenaventura). Para los otros casos analizados (Bogotá, Barranquilla y Medellín) el aporte subraya el contexto social de los pobres urbanos, esa categoría sin rostro, sin género y sin sexo que sigue rondando las ciencias sociales en América Latina. No se alega que raza sea una categoría auto-explicativa o que sea obligatoria en estos contextos, pero 
los marcadores lingüísticos/espaciales no dejan duda sobre el papel del racismo en estos escenarios de violencia. Uno tendría que preguntarse, por ejemplo, acerca de la demografía urbana de Cazucá, Ciudad Bolívar (Cárdenas, 2018), o por qué «los llamados negritos» en Barranquilla pasaron de «simple pandilla a gran estructura criminal». Si es cierto que la «banda de los negros» en Barranquilla es irrelevante para una asociación entre ser negro y criminal, es posible que este marcador lingüístico nos diga algo sobre cómo raza, segregación espacial, disputas territoriales y violencia macroeconómica se articulan en el espacio urbano de la «Puerta de Oro» de Colombia. En algunos casos, la categoría raza aparece como un aspecto descriptivo, sin ninguna consecuencia epistémica para el análisis de la violencia. Aquí es donde el concepto de escenarios y campos de violencia me parece sub-aprovechado, no haciendo justicia a Guzmán y Camacho.

¿Cuáles son las relaciones entre el desarrollo histórico de determinados órdenes sociales y escenarios de violencia en contextos fuertemente racializados como Cali y Buenaventura? ¿Cuáles las diferencias que se establecen en las dinámicas de la violencia urbana (y respuestas estatales a ellas) en ciudades con expresiva población negra y en ciudades históricamente pensadas como blanco/mestizas como Bogotá y Medellín? ¿Qué tipo de orden social se establece en un régimen racializado de ciudadanía? Mientras para los casos de Medellín, Bogotá y Barranquilla las perspectivas de raza y etnicidad se omiten del debate, en los otros dos escenarios hay una discusión de dichas categorías sin considerar las maneras como se articulan en la producción de la ciudad y de sus distintas formas de control. Una cosa es el diagnóstico de las vulnerabilidades históricas de las poblaciones negras, las mujeres y otras minorías sociales. Otra cosa bien distinta es considerar la economía política que se establece a partir de dichas manifestaciones de violencia. Si bien el papel del racismo en la victimización de ciertos grupos es obvio, menos obvio es el papel de la raza en la producción de dicho orden. Lo mismo pude ser dicho sobre «género» como categoría de análisis. El tema del «feminicidio» sobresale en el análisis de Álvaro Guzmán, Alba Nubia Pizarro y Nathalia Muñoz sobre la violencia en Cali. Estos autores/as en verdad demuestran cómo el orden paramafioso produce los cuerpos femeninos como cuerpos desechables y cómo esta producción de la alteridad femenina brutalizada sostiene el régimen de control patriarcal. Lo mismo, sin embargo, no aparece en los análisis sobre las otras urbes. Más allá de diagnosticar la victimización femenina, habría que preguntarse cómo la violencia de género se articula con la economía y el orden político de las ciudades. Los trabajos de autoras como Danny Ramírez Torres (2017) y Betty Ruth Lozano Lerma (2016) para el caso de Buenaventura, y Rita Segato (2013) para el caso de la frontera México-Estados Unidos, nos ayudan a entender cómo cuerpos racializados, feminizados, sexualizados, casas de pique, 
cuerpos descuartizados y espectáculos de muerte fundan un tipo específico de soberanía, lo que Segato llama de «segundo estado». Para esta autora, en el régimen patriarcal y orden mafioso de Ciudad Juárez, el cuerpo asesinado de las mujeres es un índice de soberanía, un índice de la alteridad masculina y de su comunidad imaginada. Los cuerpos de las muertas de Juárez, así como las «mariposas» (como son llamadas las víctimas de feminicidio en Buenaventura), son territorios-símbolo de reafirmación del poder del Estado patriarcal y su orden socioespacial. Podríamos, entonces, hablar de una economía política de la muerte en estos contextos urbanos. Otra vez, la muerte presenta su dimensión funcional/productiva.

En el lanzamiento del libro en Cali, Álvaro Guzmán rindió un homenaje a Álvaro Camacho, quien falleció de manera repentina antes de la realización del proyecto. Fiel a una tradición académica comprometida con la búsqueda de respuestas a los problemas de la sociedad colombiana, Guzmán también lamentó la falta de tiempo y de recursos para socializar los hallazgos con las organizaciones comunitarias. Leyendo el libro, el/la lectora no tendrá duda de la contribución sociológica inaugurada con los dos Álvaros en el tratamiento de un tema tan importante para Colombia. Tampoco tendrá dudas sobre las bases ético-humanistas que dieron forma a la investigación en contextos tan distintos y a la vez tan cercanos. Violencia en cinco ciudades colombianas es lectura obligatoria por el rigor académico, por los caminos que abre a nuevas inquietudes y por su relevancia política para el contexto urbano colombiano en un momento crítico donde la «paz negativa» es incierta y la «paz positiva» sigue siendo una utopía.

\section{Referencias}

ALVES, J. (2017). Gubernamentalidad espacial y agencia criminal negra en Cali y São Paulo: aproximaciones para una antropología «fuera de la ley». En J. Giraldo (coord.), Territorio y sociabilidades violentas. Santo Domingo, San Juan, Sao Paulo, Cali y Medellín (pp. 41-76.). Medellín: Universidad Eafit.

ALVES, J. (2014). Refusing to be Governed. State Delinquency Gang Violence and the Politics of Evilness in an Afrocolombian Shantytown. Manuscrito inédito.

BOURGOIS, P. (2003). In Search of Respect: Selling Crack in El Barrio. Cambridge: Cambridge University Press.

BROTHERTON, D. (2008). Beyond social Reproduction: Bringing Resistance Back in Gang Theory. Theoretical Criminology, 12 (1), 55-77. 
CÁRDENAS, R. (20108). 'Thanks to my Forced Displacement': Blackness and the Politics of Colombia's War Victims. Latin American and Caribbean Ethnic Studies, 13 (1), 72-93.

GUTIÉRREZ RIVERA, L. (2010). Discipline and Punish? Youth Gangs' Response to 'Zero Tolerance' Policies in Honduras. Bulletin of Latin American Research, 29 (4), 492-504.

LERMA, B. (2016). Violencias contra las mujeres negras: neo conquista y neo colonización de territorios y cuerpos en la región del Pacífico colombiano. La manzana de la discordia, 11 (1), 7-17.

RAMÍREZ, M. (2001). Entre el Estado y la guerrilla. Identidad y ciudadanía en el movimiento de los campesinos cocaleros del Putumayo. Bogotá: Instituto Colombiano de Antropología e Historia/ Colciencias.

RAMÍREZ TORRES, D. (2017). Feminicidios en las economías criminales de Buenaventura. Una perspectiva desde el territorio y las relaciones de género (Tesis doctoral). Universidad Nacional, Bogotá, Colombia.

ROGERS, D. (2006). The State as a Gang. Critique of Anthropology, 26 (3), 315-330.

SEGATO, R. (2013). La escritura en el cuerpo de las mujeres asesinadas en Ciudad Juárez. Territorio, soberanía y crimenes de segundo estado. Buenos Aires: Tinta Limón.

SCOTT, J. (1986). Everyday forms of peasant resistance. The Journal of Peasant Studies, 13 (2), 5-35.

TILLY, C. (1985). War Making and State Making as Organized Crime. En P. Evans, D. Rueschemeyer y T. Skocpol (eds.), Bringing the State Back In (pp. 35-6o). Cambridge: Cambridge University Press.

VALENCIA, S. (2012). Capitalismo gore y necropolítica en México contemporáneo. Relaciones internacionales, (19), 83-102.

VALVERDE, J. (2015). Narrativas mágico-religiosas en las pandillas. Un estudio sobre la psicología del pandillero. Revista CS, (17), 133-160. 